\title{
Rastreamento, Diagnóstico e Tratamento das Lesões Precursoras e do Câncer Invasor de Colo Uterino no Município de Franco da Rocha, SP
}

\author{
Screening, Diagnosis and Treatment of Precursory Lesions and Invasive Cervi- \\ cal Cancer in the City of Franco da Rocha, São Paulo, Brazil
}

Renata Guarisi', Ellen Hardy², Sophie Françoise Mauricette Derchain 3 , Gislaine Aparecida Fonsechi-Carvasan ${ }^{4}$ e João Bosco Ramos Borges ${ }^{5}$

\section{Resumo}

O objetivo deste estudo do tipo transversal foi avaliar a gravidade da lesão cervical entre mulheres com biópsia de colo uterino compatível com lesão precursora ou câncer e os principais fatores associados ao diagnóstico histológico, bem como os procedimentos e materiais disponíveis para rastreamento, diagnóstico e tratamento. Foram revisados os prontuários de todas as mulheres com biópsia de colo uterino alterada atendidas no Hospital Regional de Franco da Rocha, entre janeiro de 1998 e dezembro de 2002. Os recursos existentes no hospital e nos postos de saúde do Município foram avaliados através de entrevista com um funcionário de cada local. A cobertura do exame de citologia (CO) foi calculada embasada nos dados do Datasus. Para a análise da associação entre as variáveis e o diagnóstico histológico foram calculados os Odds Ratio com intervalo de confiança de $95 \%$. Foram identificados 87 prontuários: 52\% HPV/ NIC 1, 27\% NIC 2/ NIC 3, e 21\% câncer invasor. As variáveis associadas ao diagnóstico de NIC 2/ NIC 3 foram procura de atendimento por CO ou biópsia alterada, idade de 35 a 49 anos e ter tido cinco partos ou mais. Por outro lado, procura de atendimento espontaneamente e por sintoma específico de câncer e, idade de 35 a 49 anos e 50 anos ou mais, ter tido cinco partos ou mais, ou o primeiro parto com 18 anos ou mais, não usar anticoncepcional hormonal e não possuir CO prévia foram variáveis associadas ao câncer invasor. A cobertura do exame citológico foi menor que $25 \%$ em todos os anos. Palavras-chave: neoplasias do colo uterino; condiçôes pré-cancerosas; citodiagnóstico; rastreamento.

\footnotetext{
${ }^{1}$ Mestre em Tocoginecologia, Unicamp. Professora Colaboradora, Disciplina de Ginecologia, Faculdade de Medicina de Jundiaí

${ }^{2}$ Doutora em Saúde Pública. Professora Associada, Depto. de Tocoginecologia, Unicamp

${ }^{3}$ Livre Docente em Tocoginecologia, Unicamp. Professora Associada, Depto. de Tocoginecologia, Unicamp

${ }^{4}$ Mestre em Saúde Coletiva, Departamento de Tocoginecologia, Unicamp

${ }^{5}$ Doutor em Tocoginecologia, Universidade de São Paulo. Professor Adjunto, Disciplina de Ginecologia, Faculdade de Medicina de Jundiaí Instituição: Universidade Estadual de Campinas (Unicamp) - Endereço para correspondência: Renata Guarisi - Rua José Perez, 120 - Jardim Pacaembú - 13.218.202 Jundiaí, SP

Trabalho parcialmente financiado pela FAPESP Processo no 03 / 03601 - 3
} 


\section{Abstract}

The objective of this cross-sectional study was to evaluate the severity of cervical lesion among women with preneoplasic or invasive cervical biopsy, the characterisitics of these women, as well as the procedure and resources available to screen, diagnose and treat them. The files of all women with abnormal cervical histological diagnosis, who attended the Franco da Rocha Regional Hospital between 1998 and 2002, were reviewed. To evaluate the resources available in the hospital and in the primary healthcare units, an interview was carried out with one employee from each facility. The Pap test coverage was estimated using data from Datasus. Odds Ratios, with a confidence interval of $95 \%$, were estimated for the analysis of the association between the variables and the histological diagnosis. Eighty-seven files were identified - 52\% with a diagnosis of HPV / CIN 1, 28\% of CIN 2 / CIN 3, and 21\% of invasive cervical cancer. Presenting to the hospital because of abnormal cytology and/or biopsy results, being between 35 and 49 years old and having had five or more deliveries were factors associated with CIN 2 / CIN 3. Presenting for a specific symptom of cancer, attending the hospital spontaneously, being 50 or more years old, or 35 to 49 years of age, having had five or more deliveries, not having used contraceptive pills and not having had a previous Pap smear were factors positively associated with a diagnosis of invasive neoplasia. The Pap smear coverage was below $25 \%$ for every year studied.

Key words: cervix neoplasms; precancerous conditions; cytodiagnosis; screening.

\section{INTRODUÇÃO}

O câncer invasor do colo uterino continua sendo o segundo câncer mais freqüente entre mulheres no mundo e o mais comum em países em desenvolvimento. ${ }^{1}$ Segundo a Organização Mundial da Saúde (OMS), a cada ano são diagnosticados 470.000 novos casos de câncer cervical, e ocorrem aproximadamente 230.000 mortes, sendo cerca de $80 \%$ delas em países em desenvolvimento. ${ }^{1}$ No Brasil é a neoplasia maligna que mais acomete o trato genital feminino, ${ }^{2}$ apresentando estimativas de incidência e mortalidade para o ano de 2003 de 18,3/100.000 e 4,6/100.000 mulheres, respectivamente ${ }^{3}$, constituindo-se um grande problema de saúde pública. ${ }^{4,5}$ Baixo nível socioeconômico e cultural, idade precoce ao início de atividade sexual, múltiplos parceiros, multiparidade e tabagismo são fatores classicamente descritos como predisponentes para esta neoplasia. ${ }^{1,67,8} \mathrm{O}$ principal agente promotor da neoplasia cervical é o Papilomavírus Humano (HPV) que é, atualmente, a doença sexualmente transmissível (DST) mais freqüente. ${ }^{9,10}$

O câncer cervical é uma doença passível de prevenção, que pode ser efetuada através da erradicação do HPV ou da detecção e tratamento das lesões precursoras. ${ }^{11,12}$ Estudos clínicos de vacinas anti-HPV estão em desenvolvimento e a erradicação do HPV encontra-se em fase de experimentação., ${ }^{34}$ Por outro lado, a detecção e tratamento das lesões precursoras é um método viável e eficaz na prevenção do câncer de colo uterino. Entretanto, para que o diagnóstico precoce seja realizado com êxito, são necessários programas de rastreamento organizados, já implementados com sucesso em muitos países, onde se observou uma redução significativa (70\%) na incidência e mortalidade por câncer cervical. . $^{2,510,14}$ Os programas de rastreamento devem ter uma alta efetividade e o menor custo possível. ${ }^{16}$ Os programas que tentam incluir todas as mulheres através de convites objetivos e personalizados mostram-se mais eficientes do que aqueles em que os controles são realizados por demanda espontânea. ${ }^{17} \mathrm{~A}$ proposta destes programas inclui diagnosticar e tratar lesōes de alto grau, prevenindo a progressão para a forma invasora. ${ }^{18} \mathrm{~A}$ maioria deles baseia-se na aplicação do exame de Papanicolaou ou citologia oncológica (CO). ${ }^{2,10,19}$ No entanto, esses programas não foram implantados na maioria dos países em desenvolvimento, nos quais os exames são realizados por demanda espontânea e conseqüentemente a cobertura populacional é baixa.

Os obstáculos ao estabelecimento de tais programas são muito grandes, incluindo custos, processos educativos e interesses políticos. ${ }^{25,10}$ No Brasil, foram implementadas açōes governamentais e individuais, nas últimas décadas, com o objetivo de aumentar a cobertura populacional do exame de Papanicolaou. Um dos primeiros programas implantados no Estado de São Paulo foi o de Campinas, iniciado em 1968, com redução significativa na detecção do câncer cervical avançado. ${ }^{20,21} \mathrm{O}$ Ministério da Saúde, através do Instituto Nacional de Câncer (INCA), lançou o Programa Viva Mulher em março de 1996. O objetivo do Programa é diminuir a incidência e mortalidade por câncer de colo do útero, através da ampliação do acesso das mulheres ao exame de Papanicolaou, priorizando as de maior risco, e garantindo diagnóstico, tratamento e seguimento adequados. ${ }^{22}$

As medidas desenvolvidas pelo Programa deveriam 
atingir todas as localidades do país; entretanto, não há informaçōes com relação a sua implantação em muitos municípios. Franco da Rocha, situado a $30 \mathrm{~km}$ da capital do Estado de São Paulo, é um exemplo. Apesar de estar na Região Sudeste, onde são encontradas as menores taxas de incidência de câncer de colo no Brasil, a população de Franco da Rocha apresenta características de vida muito precárias. Predominam neste Município mulheres de baixos níveis socioeconômico e cultural, condições que estão altamente relacionadas ao câncer cervical. Esses baixos níveis têm sido avaliados pelas condições de vida no Estado de São Paulo, segundo o Índice Paulista de Responsabilidade Social (IPRS). Este índice inclui as seguintes categorias: dimensão de riqueza, longevidade e escolaridade. Os valores das categorias para o Estado foram de 60, 60 e 71, respectivamente, em 1997. Neste mesmo ano, Franco da Rocha apresentou índices bem menores: 47, $52 \mathrm{e}$ 58, respectivamente. ${ }^{31}$ Em 2002, Franco da Rocha possuía uma população total de 112.870 habitantes, com 54.439 mulheres. Dessas, 36.854 tinham entre 15 e 69 anos. ${ }^{23} \mathrm{~A}$ única informação encontrada a respeito da prevenção do câncer de colo é a de Hardy et al. (1989) ${ }^{24}$, que observaram que apenas $53,7 \%$ de 339 mulheres entrevistadas em Caieiras, Franco da Rocha e Francisco Morato haviam feito o exame de Papanicolaou pelo menos uma vez. Em Franco da Rocha não se conhece a verdadeira cobertura obtida com a $\mathrm{CO}$, a proporção de mulheres com exames alterados, nem se são efetivamente tratadas e acompanhadas. Também não se sabe se os recursos para a realização do controle do câncer cervical são suficientes e bem utilizados.

Desta forma, este estudo teve como objetivo avaliar a gravidade da lesão cervical entre as mulheres com biópsia de colo uterino compatível com lesão precursora ou câncer, os principais fatores associados ao diagnóstico histológico, os procedimentos e materiais disponíveis para rastreamento, diagnóstico e tratamento no único hospital público (Hospital Regional de Franco da Rocha) e nos três postos de saúde da região, bem como estimar a cobertura do exame de Papanicolaou no município entre 1998 e 2002.

\section{SUJEITOS E MÉTODOS}

O Hospital de Franco da Rocha é referência para as unidades de saúde municipais e de outras cidades pertencentes à Divisão Regional da Saúde (DIR) IV: Caieiras, Cajamar, Francisco Morato, Mairiporã. No ano de 2000, o Serviço Municipal contava com três postos de saúde ou Unidades Básicas de Saúde (UBS): Dr. Franco da Rocha, Dr. Leopoldo José Passos e Dr. Osório César. Além das UBS existiam cinco equipes do
Programa de Saúde da Família (PSF). Para este estudo foram avaliados os postos e o Hospital, através do Ambulatório de Especialidades.

Foi realizado um estudo de corte transversal, com componentes descritivos e analíticos. O projeto foi aprovado pelo Comitê de Ética em Pesquisa da Universidade Estadual de Campinas (Unicamp). A casuística foi identificada a partir de arquivos e prontuários existentes no Hospital Regional de Franco da Rocha. Os prontuários de todas as mulheres que apresentaram biópsia de colo uterino compatível com HPV, NIC 1, NIC 2, NIC 3 e câncer cervical invasor, atendidas durante o período estudado, foram identificados através do livro de registro de biópsias no Laboratório de Anatomia Patológica do Hospital. Estes prontuários foram verificados registrando, em ficha específica, as informaçoos referentes ao motivo do encaminhamento e demanda, às características sociodemográficas, reprodutivas e sexuais das mulheres, e ao diagnóstico, tratamento e seguimento.

A cobertura do exame de Papanicolaou foi estimada através dos dados do Datasus, onde foi identificado o número de mulheres entre 15 e 69 anos, residentes em Franco da Rocha em cada ano estudado, e o número de CO coletadas no município entre 1998 e 2002. Para a avaliação dos recursos humanos e materiais disponíveis para o rastreamento e diagnóstico nos postos de saúde em 2002, foi enviada uma carta à Diretora de Saúde solicitando permissão para o acesso aos arquivos e prontuários e a realização de entrevista com um funcionário de cada posto. Enfermeiros dos postos de saúde e do hospital, que conheciam a rotina de atendimento do local, foram convidados a participar do estudo. Eles foram informados a respeito dos objetivos do mesmo e do seu direito de não participação. Assinaram o Termo de Consentimento Livre e Esclarecido ao aceitar participar. ${ }^{25,26}$ Para a entrevista utilizou-se questionário especifico. A coleta dos dados e as entrevistas foram feitas pela pesquisadora principal, de julho de 2002 a janeiro de 2003.

Para a análise da associação entre as variáveis e o diagnóstico histológico foram calculados os Odds Ratio com intervalo de confiança de $95 \%$. As variáveis estudadas foram: motivo do encaminhamento, demanda, local de moradia e nascimento, idade ao diagnóstico, cor, estado marital, tabagismo, idade ao início da atividade sexual, número de parceiros sexuais, idade ao primeiro parto, número de partos, antecedente de DST, exame citológico prévio e uso de contraceptivo hormonal. Os diagnósticos foram divididos em três grupos: HPV / NIC 1, NIC 2 / NIC 3 e câncer invasor. O grupo HPV / NIC 1 foi usado como referência para a análise. 


\section{RESULTADOS}

\section{a) Distribuição dos diagnósticos e associação entre as variáveis}

Foram identificados 87 prontuários de mulheres com lesão pré-neoplásica ou neoplásica do colo do útero, sendo que $52 \%$ dos diagnósticos eram compatíveis com HPV / NIC 1, 27\% com NIC 2 / NIC 3, e 21\% com câncer cervical invasor (Tabela 1).

Entre as mulheres com NIC 2/ NIC 3, 19 procuraram o hospital por apresentar $\mathrm{CO}$ e/ou biópsia alteradas, enquanto aquelas com HPV/ NIC 1 ou câncer invasor chegaram por outros motivos. A procura de atendimento por sintoma de câncer esteve associada positivamente com o diagnóstico de câncer invasor. Com relação à demanda, observou-se que $62(90 \%)$ entre as 69 mulheres com lesão precursora chegaram ao hospital encaminhadas de outro serviço. Ao contrário, mais da metade daquelas com câncer invasor procuraram o hospital espontaneamente (Tabela 2).

Tabela 1. Distribuição percentual dos diagnósticos segundo o ano

\begin{tabular}{l|c|c|c|c|c|c}
\hline \multicolumn{7}{|c}{ Ano do diagnóstico (\%) } \\
\hline Diagnóstico & 1998 & 1999 & 2000 & 2001 & 2002 & Total \\
\hline HPV/ NIC 1 & 31 & 63 & 62 & 65 & 43 & 52 \\
\hline NIC 2/ NIC 3 & 54 & 12 & 23 & 22 & 27 & 27 \\
\hline Câncer invasor & 15 & 25 & 15 & 13 & 30 & 21 \\
\hline Número total de mulheres (n) & $(13)$ & $(8)$ & $(13)$ & $(23)$ & $(30)$ & $(87)$ \\
\hline
\end{tabular}

Tabela 2. Motivo do encaminhamento e demanda de acordo com o diagnóstico histológico

\begin{tabular}{l|c|c|c|c|c}
\hline & \multicolumn{3}{|c|}{ Diagnóstico (n) } & \multicolumn{2}{c}{ Odds Ratio (IC 95\%) } \\
& $\begin{array}{l}\text { HPV / } \\
\text { NIC 1 }\end{array}$ & $\begin{array}{c}\text { NIC 2/ } \\
\text { NIC 3 }\end{array}$ & $\begin{array}{c}\text { Câncer } \\
\text { invasor }\end{array}$ & NIC 2/ NIC 3 & Câncer invasor \\
\hline CO ou Bi alterada & 22 & 19 & 03 & Ref. & Ref. \\
\hline Outro motivo: & 23 & 05 & 15 & $0,25(0,08$ a 0,79) & $4,78(1,21$ a 18,83) \\
\hline 1. Sintoma de câncer & 05 & 02 & 11 & $0,46(0,04 a 3,29)$ & $16,13(2,66$ a 23,27) \\
\hline 2. Patologia benigna & 10 & 03 & 04 & $0,35(0,05$ a 1,65) & $2,93(0,40$ a 23,27) \\
\hline 3. Verruga vulvar & 07 & 0 & 0 & Não calculável & Não calculável \\
\hline 4. Marido com HPV & 01 & 0 & 0 & Não calculável & Não calculável \\
\hline Número total & 45 & 24 & 18 & & \\
\hline
\end{tabular}

\begin{tabular}{l|c|c|c|c|c}
\hline Demanda & & & & & \\
\hline Referenciada & 38 & 24 & 8 & Ref. & Ref. \\
\hline Espontânea & 7 & 0 & 10 & Não calculável & $6,79(1,70$ a 28,38) \\
\hline Número total & 45 & 24 & 18 & & \\
\hline
\end{tabular}

Bi: biópsia; Ref: referência

Mais de $90 \%$ das mulheres, independentemente do diagnóstico, eram residentes em um dos cinco municípios da DIR IV. Contudo, observou-se uma alta taxa de migração, pois mais de $2 / 3$ das mulheres eram nascidas em outros locais. A maior taxa de migração foi a dos demais municípios do Sudeste (40\%), seguida pelos do Nordeste (25\%) do Brasil (Tabela 3).

As mulheres eram, na sua maioria, casadas (58\%) e não brancas $(63 \%)$, e um terço delas era tabagista. Quando se compararam os grupos com relação ao estado marital, cor, idade ao iniciar atividade sexual, número de parceiros, antecedente de DST e tabagismo não foi encontrada nenhuma associação com o diagnóstico histológico (informações não mostradas em tabela).

Sessenta e nove por cento dos diagnósticos de HPV/ NIC 1 foram feitos em mulheres de até 35 anos de idade. Os casos de câncer invasor foram diagnosticados, em $89 \%$ das vezes, depois desta idade. Já os casos de NIC 2/ NIC 3 ocorreram em uma idade intermediária. Deste modo, as faixas etárias de 50 ou mais e 35 a 49 anos associaram-se positivamente ao diagnóstico de câncer invasor, e apenas esta última mostrou associação positiva ao diagnóstico de NIC 2 /NIC 3 . Ter tido o primeiro parto com 18 anos ou mais, não ter usado 
anticoncepcional hormonal e não ter realizado $\mathrm{CO}$ anteriormente estiveram associados positivamente com o diagnóstico de câncer invasor. Ter tido cinco partos ou mais foi a única variável associada tanto com o diagnóstico de NIC 2/ NIC 3 quanto com o de câncer invasor (Tabela 4).

Tabela 3. Locais de moradia e de nascimento segundo o diagnóstico

\begin{tabular}{|c|c|c|c|}
\hline & \multicolumn{3}{|c|}{ Diagnóstico (\%) } \\
\hline Local & HPV / NIC 1 & $\mathrm{NIC} 2 / \mathrm{NIC} 3$ & Câncer invasor \\
\hline \multicolumn{4}{|l|}{ Moradia* } \\
\hline DIR IV & 95 & 92 & 94 \\
\hline Outros municípios & 5 & 8 & 6 \\
\hline Número total (n) & (43) & $(24)$ & (18) \\
\hline \multicolumn{4}{|l|}{ Nascimento* } \\
\hline DIR IV & 23 & 30 & 31 \\
\hline Nordeste & 30 & 35 & 19 \\
\hline Centro-Oeste & 3 & - & - \\
\hline Sudeste & 40 & 35 & 44 \\
\hline Sul & 3 & - & 6 \\
\hline Número total (n) & (30) & (17) & (16) \\
\hline
\end{tabular}

${ }^{*}$ Faltou informação: moradia $=2$; nascimento $=24$

Tabela 4. Odds ratio das características das mulheres segundo o diagnóstico

\begin{tabular}{|c|c|c|c|c|c|}
\hline \multirow[b]{2}{*}{ Características* } & \multicolumn{3}{|c|}{ Diagnóstico (n) } & \multicolumn{2}{|c|}{ Odds Ratio (IC 95\%) } \\
\hline & $\begin{array}{l}\text { HPV / } \\
\text { NIC } 1\end{array}$ & $\begin{array}{l}\text { NIC 2/ } \\
\text { NIC } 3\end{array}$ & $\begin{array}{l}\text { Câncer } \\
\text { invasor }\end{array}$ & NIC 2/ NIC 3 & Câncer invasor \\
\hline \multicolumn{6}{|l|}{ Idade (em anos) } \\
\hline$<35$ & 31 & 10 & 02 & Ref. & Ref. \\
\hline 35 a 49 & 09 & 10 & 08 & $3,40(1,09$ a 10,86$)$ & $13,78(2,47$ a 76,79$)$ \\
\hline 50 ou + & 05 & 04 & 08 & $2,48(0,56$ a 11,07$)$ & $24,8(4,04$ a 152,26$)$ \\
\hline Total & 45 & 24 & 18 & & \\
\hline \multicolumn{6}{|c|}{ Idade no $1^{\circ}$ parto $\left(\right.$ anos) ${ }^{* *}$} \\
\hline$<18$ & 16 & 09 & 02 & Ref. & Ref. \\
\hline 18 ou + & 23 & 09 & 16 & $0,70(0,20$ a 2,49$)$ & $5,57(1,04$ a 55,13$)$ \\
\hline Total & 39 & 18 & 18 & & \\
\hline \multicolumn{6}{|l|}{$\mathrm{N}^{\circ}$ de partos** } \\
\hline$<5$ & 36 & 11 & 08 & Ref. & Ref. \\
\hline 5 ou + & 03 & 10 & 10 & $10,91(2,18$ a 69,19$)$ & $15,00(2,82$ a 97,92$)$ \\
\hline Total & 39 & 21 & 18 & & \\
\hline \multicolumn{6}{|l|}{$\mathrm{CO}$ anterior } \\
\hline Sim & 31 & 15 & 07 & Ref. & Ref. \\
\hline Não & 05 & 02 & 06 & $0,83(0,14$ a 4,77$)$ & $5,31(1,26$ a 22,49$)$ \\
\hline Total & 36 & 17 & 13 & & \\
\hline \multicolumn{6}{|l|}{ Uso de $\mathrm{ACH}$} \\
\hline Sim & 25 & 12 & 05 & Ref. & Ref. \\
\hline Não & 17 & 11 & 13 & $1,35(0,43$ a 4,25$)$ & $3,82(1,01$ a 16,00$)$ \\
\hline Total & 42 & 23 & 18 & & \\
\hline
\end{tabular}

*Faltou informação: idade no primeiro parto $=3$; uso de $\mathrm{ACH}=4$;

** Somente mulheres que tiveram partos 


\section{b) Diagnóstico, tratamento e seguimento}

Entre as mulheres com diagnóstico hitopatológico de HPV / NIC 1, pouco mais da metade (55\%) apresentavam CO normal. Já entre aquelas com NIC 2 / NIC 3 e câncer invasor esta porcentagem foi de $21 \%$ e $25 \%$, respectivamente. O diagnóstico definitivo foi feito através de colposcopia e biópsia dirigida em 100\% das mulheres com HPV / NIC 1, 92\% das com NIC 2/ NIC 3 e 95\% das com câncer invasor. Em duas mulheres com NIC 2/ NIC 3 e uma com câncer invasor o diagnóstico ocorreu pós-histerectomia, indicada por outros motivos (informaçôes não mostradas em tabela).

$\mathrm{O}$ tratamento realizado para mais da metade das mulheres com diagnóstico de HPV / NIC 1 foi apenas seguimento com CO e colposcopia. Vinte das 24 mulheres com NIC 2/ NIC 3 foram submetidas à conização uterina e duas à histerectomia. Uma mulher com biópsia compatível com NIC 3 foi submetida à conização, mas a avaliação histológica da peça não determinou se havia ou não invasão, e por este motivo, foi submetida à cirurgia de Wertheim-Meigs. A metade das mulheres com câncer invasor foi submetida à radioterapia exclusiva. Independente do diagnóstico histológico, a grande maioria das mulheres compareceu a todas as consultas agendadas, realizando seguimento adequado pós-tratamento. Uma pequena parte não iniciou ou interrompeu o seguimento (Tabela 5).

Tabela 5. Tratamento e seguimento recebido pelas mulheres segundo o diagnóstico

\begin{tabular}{|c|c|c|c|}
\hline & \multicolumn{3}{|c|}{ Diagnóstico (\%) } \\
\hline Tratamento* & $\mathrm{HPV} / \mathrm{NIC} 1$ & NIC 2/ NIC 3 & Câncer invasor \\
\hline Seguimento & 56 & - & - \\
\hline Cauterização & 33 & - & - \\
\hline Conização & 11 & 83 & - \\
\hline Histerectomia & - & 13 & - \\
\hline Wertheim-Meigs & - & 4 & 25 \\
\hline Cirurgia + Rxt & - & & 31 \\
\hline Rxt exclusiva & - & & 44 \\
\hline Número total (n) & (43) & (23) & (16) \\
\hline \multicolumn{4}{|l|}{ Seguimento } \\
\hline Adequado & 80 & 87 & 82 \\
\hline Inadequado & 02 & - & - \\
\hline Interrompeu & 14 & 09 & - \\
\hline Nunca iniciou & 04 & 04 & 18 \\
\hline Número total (n) & (44) & (24) & (18) \\
\hline
\end{tabular}

* Três mulheres não retornaram ao hospital para tratamento: HPV/NIC $1=1$, NIC 2/NIC 3 = 1; câncer invasor = 1 ; e uma mulher com câncer invasor foi transferida e realizou tratamento em outro hospital.

\section{c) Cobertura estimada do exame de Papanicolaou}

A cobertura do exame de Papanicolaou foi muito baixa em todos os anos estudados. A maior taxa foi observada em 2001, mesmo assim ficando abaixo dos 25\%. Em 2000, a cobertura foi ainda mais baixa, em torno de $16 \%$. A menor cobertura dos quatro anos foi observada em 1999 (Tabela 6).

\section{d) Recursos disponíveis}

Cada um dos três postos de saúde visitados possuía dois ginecologistas e uma ou duas enfermeiras. O tempo de espera para consulta com ginecologista era de 30 a 60 dias em todos os postos. Em um deles, o exame de Papanicolaou era colhido pelo ginecologista, enfermeira ou auxiliar de enfermagem que havia sido treinada. Nos demais, a coleta era realizada apenas pelo ginecologista. Para a coleta utilizava-se espátula de Ayre e escova endocervical. As lâminas eram encaminhadas para leitura em laboratório terceirizado, e os resultados chegavam ao posto em até 30 dias. Entretanto, apenas um posto possuía registro dos resultados de $\mathrm{CO}$, outro não tinha nenhum registro, e no terceiro o registro começou em 2002 (informações não mostradas em tabela).

Todos os postos possuíam material necessário para coleta da CO. Entretanto, apenas um deles possuía ácido acético e solução de lugol para exame do colo do útero. Apenas o posto B era munido de colposcópio e demais materiais para realização do diagnóstico de lesões 
precursoras e câncer cervical. O postos A e B possuíam eletrocautério e nenhum dos três possuía CAF. Este aparelho só foi encontrado no Ambulatório de Especialidades do Hospital, que também possuía todo o material necessário para o rastreamento e diagnóstico (Quadro 1).

As mulheres com citologias alteradas eram convocadas a comparecer através de aerogramas enviados por dois postos (A e B). No outro, aguardavase a mulher comparecer ao retorno com o ginecologista. As mulheres com $\mathrm{CO}$ alterada que compareciam eram encaminhadas para complementação diagnóstica (informações não mostradas em tabela).

O Ambulatório de Especialidades funcionava como unidade de referência. Nele eram realizados o diagnóstico e tratamento das lesôes precursoras. Quando era diagnosticado câncer invasor, a mulher era tratada no próprio hospital se o tratamento indicado fosse cirúrgico. No caso de indicação de tratamento actínico, a mulher era encaminhada para o Instituto Arnaldo Vieira de Carvalho, no Município de São Paulo. O seguimento das mulheres tratadas, tanto por lesão precursora quanto por câncer invasor, era realizado no Hospital (informações não mostradas em tabela).

Tabela 6. Cobertura estimada do exame de Papanicolaou em cada ano

\begin{tabular}{c|c|c|c}
\hline Ano & $\begin{array}{c}\text { População sob risco } \\
\text { (15 a 69 anos) }\end{array}$ & $\begin{array}{c}\text { Número de } \\
\text { citologias colhidas }\end{array}$ & Cobertura estimada (\%) \\
\hline 1998 & 33.850 & 6.019 & 17,8 \\
\hline 1999 & 34.925 & 3.328 & 9,5 \\
\hline 2000 & 35.302 & 5.718 & 16,2 \\
\hline 2001 & 36.161 & 8.698 & 24,1 \\
\hline 2002 & 36.854 & 6.375 & 17,3 \\
\hline
\end{tabular}

Fonte: Datasus

Quadro 1. Facilidades existentes de acordo com o local

\begin{tabular}{|c|c|c|c|c|}
\hline \multicolumn{5}{|c|}{ Postos } \\
\hline Facilitadores para: & $A$ & B & C & Ambulatório \\
\hline Rastreamento & $X$ & $X$ & $\mathrm{X}$ & $X$ \\
\hline Luvas & $X$ & $X$ & $X$ & $X$ \\
\hline Espéculo & $X$ & $X$ & $\mathrm{X}$ & $X$ \\
\hline Espátula de Ayre & $X$ & $X$ & $X$ & $X$ \\
\hline Escova cervical & $X$ & $X$ & $X$ & $X$ \\
\hline Lâminas & $X$ & $X$ & $\mathrm{X}$ & $X$ \\
\hline Fixador & $X$ & $X$ & $X$ & $X$ \\
\hline Frascos & $X$ & $X$ & $X$ & $X$ \\
\hline \multicolumn{5}{|l|}{ Diagnóstico } \\
\hline Colposcópio & & $X$ & & $X$ \\
\hline Ácido acético & & $\mathrm{X}$ & & $X$ \\
\hline Solução de lugol & & $x$ & & $x$ \\
\hline Cheron & & $X$ & & $X$ \\
\hline Pinça de biópsia & & $X$ & & $X$ \\
\hline Formol & & $X$ & & $X$ \\
\hline \multicolumn{5}{|l|}{ Tratamento } \\
\hline Eletrocautério & $X$ & $X$ & & $X$ \\
\hline Alça para CAF & & & & $X$ \\
\hline
\end{tabular}

\section{DISCUSSÃO}

Observou-se no Hospital de Franco da Rocha que $21 \%$ das mulheres com biópsia do colo do útero alterada já apresentavam câncer de colo uterino na sua forma invasora. Este resultado sugere que o rastreamento do câncer do colo do útero neste município ainda é muito precário e necessita de implementação urgente, tendo em visa que o câncer invasor do colo uterino pode ser prevenido através da detecção e tratamento das lesões precursoras.

O presente estudo comparou grupos com diferente severidade de doença, não havendo grupo-controle sem doença, demonstrando apenas a existência ou não de associação entre as variáveis estudadas e o diagnóstico histológico. Dessa maneira, não se encontrou associação entre estado marital, número de parceiros, primeira relação sexual em idade precoce e tabagismo com a gravidade da lesão cervical, embora essas associações sejam consistentes na literatura quando se comparam mulheres sem lesão e mulheres com NIC ou câncer invasor. ${ }^{1,6}$ Com relação à idade, observou-se que até os 35 anos prevaleceu o diagnóstico de NIC 1, entre 35 e 49 anos NIC 2/ NIC 3 e câncer invasor, e a partir dos 50 anos o câncer invasor foi o diagnóstico mais prevalente. Estes resultados estáo de acordo com a história natural da doença e são coincidentes com os achados de outros autores. ${ }^{27,28}$

Chama a atenção que a maioria das mulheres com câncer invasor procurou o hospital espontaneamente e com sintoma da doença, sendo que mais da metade delas não havia realizado exame citológico previamente. As mulheres com diagnóstico de NIC 2 ou 3 chegaram referenciadas por citologia ou biópsia alterada. Estes 
dados sugerem que a $\mathrm{CO}$ teve papel fundamental na detecção de NIC 2 e 3, evitando a evolução da doença para sua forma invasora. Diferentes autores comprovaram que a realização da citologia pode reduzir a incidência deste câncer. ${ }^{14,19}$

A CO é o principal teste usado em programas de rastreamento. Mas, para haver um impacto na mortalidade, a cobertura do exame deve ser de pelo menos $70 \%$ a $80 \% .^{29} \mathrm{O}$ presente estudo mostrou uma cobertura muito baixa nos quatro anos, sempre inferior a 25\%. A menor cobertura foi observada em 1999 $(6,9 \%)$, e o motivo informado nos postos para o baixo número de exames foi a falta de material para coleta em todo o Município.

Admite-se que os resultados não são exatos por não ser um estudo de base populacional. Mesmo assim, ajudam a entender a situação, na falta de estudos recentes a respeito do rastreamento do câncer cervical neste município. A coleta da $\mathrm{CO}$ era realizada de maneira oportunista, ou seja, apenas as mulheres que procuravam o ginecologista realizavam o exame. Provavelmente as mesmas mulheres repetiam o exame anualmente. A American Cancer Society recomenda que o rastreamento comece três anos após a primeira relação sexual e no máximo até os 21 anos de idade, e que seja feito anualmente até os 30 anos. A partir de então, se a mulher apresentar dois exames consecutivos tecnicamente satisfatórios e normais, pode continuar o rastreamento a cada dois ou três anos. ${ }^{18}$

Por se basear em citologias, os programas organizados requerem laboratórios equipados e citologistas e citotécnicos bem treinados. Apesar de a $\mathrm{CO}$ ser considerada um teste para detectar lesóes de alto grau ou câncer muito específico, tanto sua especificidade quanto sensibilidade dependerão da qualidade da coleta e leitura do exame. ${ }^{30}$ Cerca de $40 \%$ das mulheres com lesão precursora ou câncer invasor possuíam CO normal. $\mathrm{Na}$ literatura, as diferenças nas taxas de resultados falsos negativos e falsos positivos são grandes, variando de $5 \%$ a $70 \%$ e de $10 \%$ a $30 \%$, respectivamente. Por este motivo, os laboratórios necessitam de rigoroso e constante controle de qualidade.

\section{SUGESTÕES}

A implantação de um programa organizado, especialmente desenvolvido para esse Município, será a melhor estratégia na tentativa de diminuir a incidência e mortalidade por câncer do colo do útero. Campanhas educacionais devem ser realizadas, visando conscientizar a população da necessidade do exame. Além disso, é necessária a elaboração de um sistema de convocação das mulheres, dando prioridade para aquelas que se encontram na faixa etária de maior risco e que nunca fizeram o exame. A convocação pode ser feita através dos agentes de saúde do Programa de Saúde da Família (PSF), sistema já implantado com sucesso pelo Ministério da Saúde.

O controle interno, com registro dos exames coletados e alterados, é imprescindível para que toda mulher com exame alterado seja submetida à investigação complementar. Através deste controle, elas são facilmente identificáveis e podem ser convocadas para complementação diagnóstica.

Com isso, espera-se um impacto positivo no rastreamento do câncer de colo nessa região diminuindo sua incidência e mortalidade.

Agradecimentos: Os autores agradecem as revisões feitas por Luiz Carlos Zeferino, Maria José Duarte Osis e a colaboração de toda a equipe do Cemicamp.

\section{REFERÊNCIAS BIBLIOGRÁFICAS}

1. Parkin DM, Bray FI, Devesa SS. Cancer burden in the year 2000. The global picture. Eur J Cancer 2001;37:S4-66.

2. Franco EL. Understanding the epidemiology of genital infection with oncogenic and nononcogenic human papillomaviruses: a promising lead for primary prevention of cervical cancer. Cancer Epidemiol Biomarkers Prev 1997;6:759-61.

3. Instituto Nacional de Câncer (Brasil). Estimativas da incidência e mortalidade por câncer no Brasil. Rio de Janeiro: INCA; 2003.

4. Syrjänen K. Natural history of low grade SIL lesions. In: Monsonego J, editor. Challenges of modern medicine. Papillomavirus in human pathology. Rome: Ares-Serono Symposia Publications; 1995. p. 97-110.

5. Miller AB, Nazeer S, Fonn S, Brandup-Lukanow A, Rehman $\mathrm{R}$, Cronje H, et al. Report on Consensus Conference on Cervical Cancer Screening and Management. Int J Cancer 2000;86:440-7.

6. Brinton LA, Fraumeni JF Jr. Epidemiology of uterine cervical cancer. J Chron Dis 1986;39:1051-64.

7. Derchain SFM, Roteli-Martins CM, Syrjänen KJ, Abreu HJ, Martinez EZ, Alves VAV. Association of oncogenic human papilomavirus (HPV) CNA with high-grade cervical intraepithelial neoplasia (CIN 2 or 3); the role of cigarette smoking. Sex Trans Inf 1999;75:406-8.

8. Kjellberg L, Hallmans G, Ahren A-M, Johansson R, Bergman F, Wadell G, et al. Smoking, diet, pregnancy and oral contraceptive use as risk factors for cervical intra-epithelial neoplasia in relation to human papillomavirus infection. Br J Cancer 2000;82:1332-8.

9. Syrjänen K, Hakama M, Saarikoski S, Väyrynen M, Yliskoski 
M, Syrjänen SO, et al. Prevalence, incidence and estimated life-time risk of cervical human papillomavirus (HPV) infections in nonselected finnish female population. Sex Transm Dis 1990;17:15-9.

10. Syrjänen S, Syrjänen K. Diagnostic techniques in HPV detection. In: Syrjänen K, Syrjänen S. Papillomavirus infections in human pathology. New York: J. Wiley \& Sons; 2000. p. 89-116.

11. Richart R, Wright T. A histologia da neoplasia do trato anogenital inferior. In: Singer A, Monaghan JM. Colposcopia, patologia e tratamento do trato genital inferior. Porto Alegre: Artes Médicas; 1995. p. 2-9.

12. Chirenje ZM, Rusakaniko S, Kirumbi L, Ngwalle EW, Makuta-Tlebere P, Kaggwa S, et al. Situation analysis for cervical cancer diagnosis and treatment in east, central and southern African countries. Bull World Health Organ 2001;79:127-32.

13. International Agency for Research on Cancer. Papillomavirus. Lyon: IARC; 1995. (Monographs on the evaluation of carcinogenic risks to humans; vol. 64).

14. Warner EA, Parsons AK. Screening and early diagnosis of gynecologic cancers. Medical Clin North Am 1996;80:45-61.

15. Miller AB. Cervical cancer screening programmes. Geneva: WHO; 1992. (Managerial guidelines).

16. Day NE. Screening for squamous cervical cancer: duration of low risk after negative results of cervical cytology and its implication for screening policies. Br Med J 1986;293:659-93.

17. Sigurdson K. Effect of organized screening on the risk of cervical cancer evalution of screening activity in Iceland. Int J Cancer 1993;54:563-70.

18. Smith RA, Cokkinides V, Eyre HJ. American Cancer Society guidelines for the early detection of cancer, 2003. CA Cancer J Clin 2003;53:27-43.

19. Mandelblatt J, Lawrence W, Gaffikin L, Limpahayom KK, Lumbiganon P, Warakamin S, et al. Costs and benefits of different strategies to screen for cervical cancer in less-developed countries. J Natl Cancer Inst 2002;94:1469-83.

20. Pinotti JA. Experiência do Programa de Controle de Câncer Cérvico-Uterino de Campinas, Brasil. J Bras Ginecol
1976;82:461-71.

21. Pinotti JÁ, Carvalho JP, Nisida ACT. Controle do câncer cervical no Brasil. Rev Ginecol Obstet 1994;5:5-10.

22. Instituto Nacional de Câncer (Brasil). Programa Nacional de Controle do Câncer do Colo do Útero e de Mama: Viva Mulher; 2000. Disponível em: http://www.inca.gov.br

23. Instituto Brasileiro de Geografia e Estatística. Censos demográficos e contagem populacional; 2002. Disponível em: http://www.datasus.gov.br

24. Hardy EE, Silva IR, Montanini LAGG, Rodrigues T, Moraes TM, Rodriguez CMR, et al. Avaliação do Programa de Assistência Integral à Saúde da Mulher no Estado de São Paulo. Relatório III. Campinas: Cemicamp;1989.

25. Declaração de Helsinke III: sobre os princípios éticos para pesquisas em seres humanos [online]. Edimburgo; 2000. Disponível em: http://www.ibemol.com.br/declaraçôes/ helsinque

26. Conselho Nacional de Saúde (Brasil). Resolução n o 196/96 sobre pesquisa envolvendo seres humanos. Bioética 1996;4:15-25.

27. Mazuelo AG, Cadena NOV. Importancia de la edad como factor de riesgo en la orientación del programa de prevención del cáncer de cuello uterino. Rev Colomb Obstet Ginecol 1996;47:185-91.

28. Luzzato L, Sfair JÁ, Omomo F, Angeli CAB, Flamia CL, Salazar C, et al. O perfil etário de pacientes com carcinomas do colo uterino: estudo em períodos cronologicamente distintos. Rev AMRIGS 1997;41:9-12.

29. Haran D, Eardley A, Elkind A, Spencer B, Smith A. Trends in the organization of cervical cancer screening. JRSH 1990;1:17-9.

30. Sankaranarayanan R, Buduck AM, Rajkumar R. Effective screening programs for cervical cancer in low- and middleincome developing countries. Bull WHO 2001;79:954-62.

31. Sistema Nacioanal de Análise de Dados (SEADE). Condiçôes de vida 1997. Disponível em: http://www.seade.gov.br

32. Goldie SJ. Health economics and cervical cancer prevention: a global perspective. Virus Res 2002;89:301-9. 